\title{
AZW-LA-II White Paint on Swift: Lessons Learned from First Time Flying on Spacecraft Radiators
}

\author{
Michael K. Choi* \\ NASA Goddard Space Flight Center, Greenbelt, MD 20771
}

\begin{abstract}
AZ-Tek's AZW-LA-II low solar absorptance white paint was previously flown as a flight experiment, and is being flown as a calorimeter. However it has never been flown as a thermal coating of radiators on an operational spacecraft before. This paint has the lowest solar absorptance among all white paints, and a very small degradation. Its cost is many times more expensive than white paints that have a higher solar absorptance. To meet the thermal requirements of the Swift Burst Alert Telescope (BAT) Detector Array and X-Ray Telescope (XRT) focal plane camera assembly (FPCA) charged coupled device (CCD), AZW-LA-II is used as the thermal coating for the BAT and XRT radiators. This paper presents the lessons learned from flying this low solar absorptance white paint as a thermal coating of radiators on an operational spacecraft for the first time.
\end{abstract}

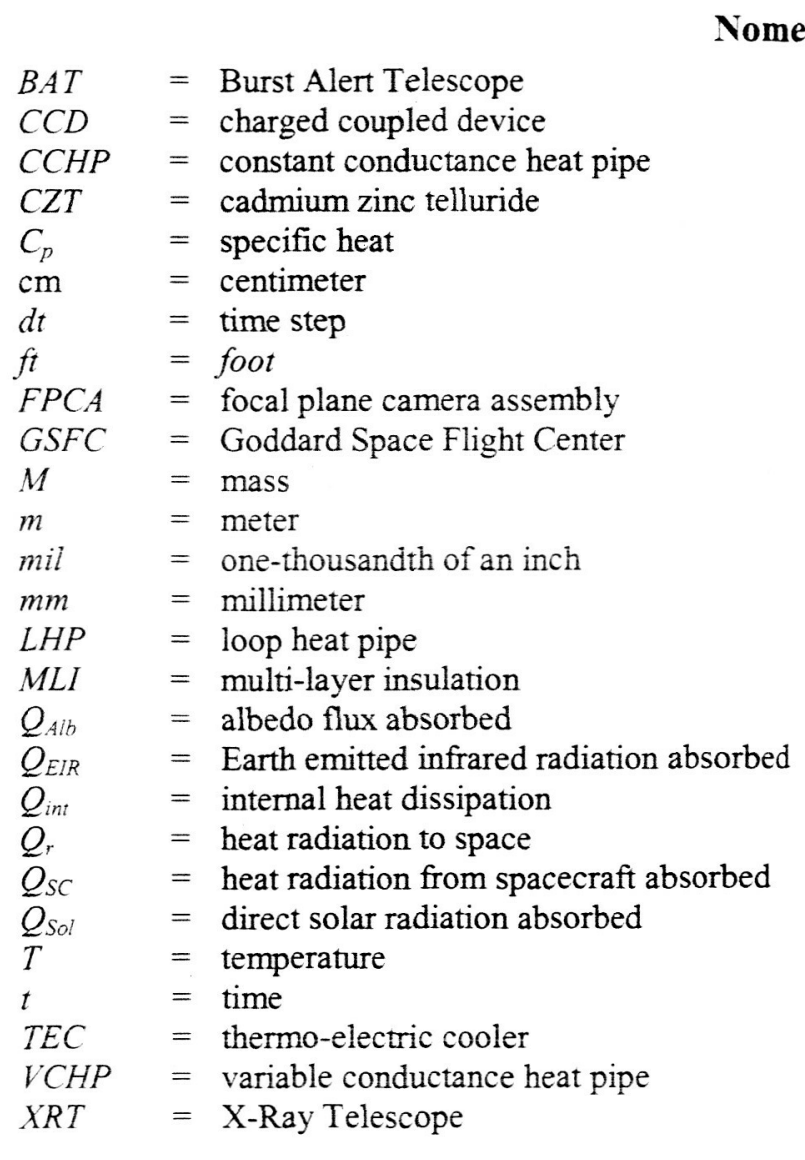

\footnotetext{
* Swift Thermal Systems Lead Engineer, Thermal Engineering Branch/Code 545, AIAA Associate Fellow.
} 


\section{Introduction}

$\mathrm{T}$ HE Swift mission is part of the National Aeronautics and Space Administration (NASA) Medium-Size Explorer (MIDEX) Program, and is managed by Goddard Space Flight Center (GSFC). The BAT is the primary instrument on the Swift spacecraft. It has been developed at GSFC. It is designed to detect gamma ray burst over a broad region of the sky in a low Earth orbit of $600-\mathrm{km}$ altitude and quickly align the telescopes on the spacecraft to the gamma ray source. Swift is scheduled to launch in October 2004. The Swift mission is a first of its kind of multiwavelength transient observatory for gamma ray burst astronomy. Its mission life is 2 years. The inclination is $22^{\circ}$ maximum.

Figure 1 shows the location of the BAT on the Swift observatory. The BAT is mounted to the optical bench through five titanium flexures. During the normal operating mode, two loop heat pipes (LHPs) transport a total of $240 \mathrm{~W}$ of thermal energy from the Detector Array to a $1.19 \mathrm{~m}(47 \mathrm{inch}) \times 1.14 \mathrm{~m}$ (44.88 inch) radiator. ${ }^{1}$ It includes $208 \mathrm{~W}$ of power dissipation of electronics and $32 \mathrm{~W}$ of operating mode heater power. Propylene is the working fluid of the LHPs. The LHP thermal system also includes two variable conductance heat pipes (VCHPs) and four header constant conductance heat pipes (CCHPs). Ammonia is the working fluid of the VCHPs and CCHPs.

$\mathrm{X}$-Ray Telescope (XRT) is one of the two narrow field instruments of the Swift observatory. Its location is also shown in Figure 1. The CCD of the XRT FPCA is cooled by a thermo-electric cooler (TEC). The waste heat of the TEC is transported by two CCHPs from the FPCA cold finger to a $1.09 \mathrm{~m}$ (43 inch) $\times 0.5588 \mathrm{~m}$ ( 22 inch) radiator. The power dissipation of the TEC is a function of the cold finger temperature $\left(3.5 \mathrm{~W}\right.$ at $-65^{\circ} \mathrm{C}, 6.5 \mathrm{~W}$ at $-50^{\circ} \mathrm{C}, 11 \mathrm{~W}$ at $-40^{\circ} \mathrm{C}, 15 \mathrm{~W}$ at $\left.-38^{\circ} \mathrm{C}\right)$. At cold finger temperatures warmer than $-38^{\circ} \mathrm{C}$ the TEC does not function as a cooler. Ethane is the working fluid of the CCHPs. ${ }^{2}$ The radiator is mounted to and thermally isolated from the spacecraft bus through four titanium flexures. The CCHPs are mounted to the cold finger through a cheater plate. Chotherm is the thermal interface material. The CCHPs, cold finger, cheater plate and radiator backside are insulated by multi-layer insulation (MLI). The parasitic heat load to the radiator is less than $2 \mathrm{~W}$.

The locations of the BAT and XRT radiators are shown in Fig. 1. Both radiators have AZ-Tek's AZW-LA-II low solar absorptance white paint as the thermal coating.

\section{Rationale of Using AZW-LA-II on BAT and XRT Radiators}

AZ-Tek's AZW-LA-II white paint was flown on the Russian's manned spacecraft MIR as a flight experiment, and is flown on NASA's unmanned spacecraft Earth Orbiter-1 (EO-1) as a calorimeter. The beginning-of-life solar absorptance measured in the flight experiment was 0.09 , which is the lowest among white paints, and the degradation due to ultraviolet radiation was very small. Also its emittance was measured to be 0.91 , which is as high as the highest value for white paints. However AZW-LA-II has never been flown as a thermal coating of radiators on an operational spacecraft before. The rationale of using this white paint is on the Swift BAT radiator and XRT radiator is as follows. The thermal requirements for the BAT detector array plane are: ${ }^{3}$

- Cadmium zinc telluride (CZT) detector operating range: a selected temperature from $0^{\circ} \mathrm{C}$ to $20^{\circ} \mathrm{C}$ in flight.

-Spatial gradient: $1^{\circ} \mathrm{C}$ maximum across entire span of $256 \mathrm{CZT}$ boards in normal operation.

-Stability: $1^{\circ} \mathrm{C}$ change maximum on any time-scale in normal operation for XA1 ASIC.

Thermal analysis, which was later validated by testing, showed that the temperature gradient between the XAl ASIC and radiator through the LHPs is $10^{\circ} \mathrm{C}$. It requires the radiator to be no warmer than $-10^{\circ} \mathrm{C}$ and stable.

The temperature requirement of the XRT FPCA CCD is $-105^{\circ} \mathrm{C}$, and the goal is $-115^{\circ} \mathrm{C}$ to provide sufficient margins to minimize the risk of radiation damage to the $\mathrm{CCD}$ in flight. $\mathrm{A}-115^{\circ} \mathrm{C} \mathrm{CCD}$ temperature translates to a temperature requirement of $-55^{\circ} \mathrm{C}$ for the cold finger. 
Albedo flux is a major factor that causes temperature instability of the BAT and XRT radiators for the following reasons:

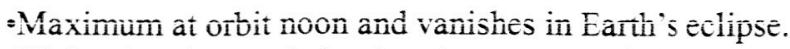

-Higher in winter solstice than in summer solstice.

-Spacecraft slews so that the sun angle changes transiently.

Figure 2 compares the environmental heat flux absorbed by AZW-LA-II and Z93P white paints when the sun angle is $90^{\circ}$ and the beta angle is $0^{\circ}$ in the Swift orbit. End-of-life thermo-optical properties and winter solstice environment are assumed. The solar absorptance of Z93P is 0.20 , and that of AZW-LA-II is 0.14 . At orbit noon the environmental heat flux absorbed by AZW-LA-II is $25 \mathrm{Wm}^{-2}$ less than that absorbed by Z93P.

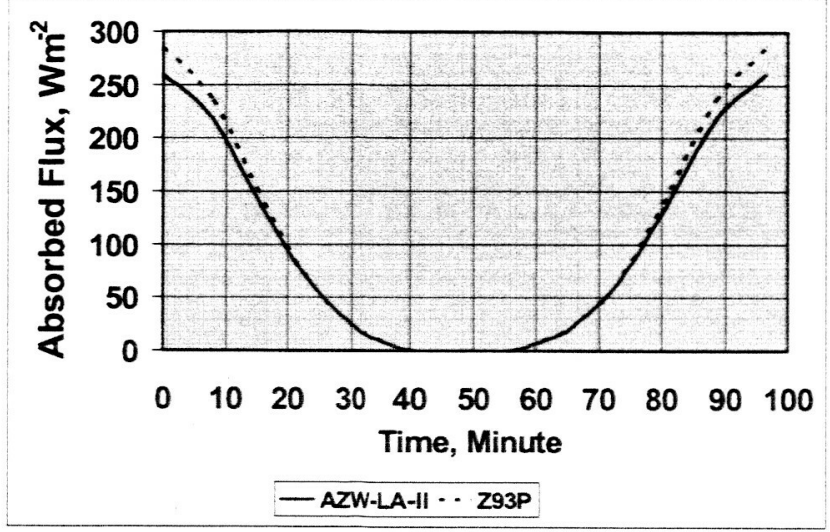

Figure 2. Comparison of Environmental Heat Flux Absorbed by AZW-LA-II and Z93P.

Thermal energy balance of a radiator in the normal operation mode is:

$$
\mathrm{Q}_{\text {Int }}(\mathrm{t})+\mathrm{Q}_{\mathrm{Alb}}(\mathrm{t})+\mathrm{Q}_{E I R}(\mathrm{t})+\mathrm{Q}_{\mathrm{SC}}(\mathrm{t})=\mathrm{Q}_{\mathrm{T}}(\mathrm{t})+\mathrm{MC}_{\mathrm{p}}(\mathrm{T}) \mathrm{dT}(\mathrm{t}) / \mathrm{dt}
$$

where $\mathrm{Qr}=$ heat radiation from radiator to space,

$M=$ mass of radiator,

$\mathrm{C}_{\mathrm{p}}=$ specific heat of radiator,

$\mathrm{Q}_{\mathrm{Int}}=$ internal power dissipation,

$\mathrm{Q}_{\mathrm{Alb}}=$ albedo flux absorbed,

$\mathrm{Q}_{\mathrm{EIR}}=$ Earth emitted infrared radiation absorbed,

$\mathrm{Q}_{\mathrm{SC}}=$ heat radiation from spacecraft absorbed.

During spacecraft slewing, direct solar radiation on the BAT and XRT radiators is allowed for up to 5 minutes. Thermal energy balance of a radiator during spacecraft slewing is:

where $\mathrm{Q}_{\mathrm{Sol}}=$ direct solar flux absorbed.

$$
Q_{\text {IInt }}(t)+Q_{A i b}(t)+Q_{E I R}(t)+Q_{S C}(t)+Q_{S o l}(t)=Q_{T}(t)+M C_{p}(T) d T(t) / d t
$$

If the solar vector is perpendicular to the radiator during a 5 minute slew and the paint is AZW-LA-II instead of Z93P, the solar flux absorbed is $71 \mathrm{Wm}^{-2}$ less. The temperature rise caused by direct solar flux is $2.5^{\circ} \mathrm{C}$ less for the $\mathrm{BAT}$ radiator and $2^{\circ} \mathrm{C}$ less for the XRT radiator.

To minimize the effect of albedo flux in normal operation and the effect of direct solar flux during slewing, so as to meet the thermal requirements, AZW-LA-II is used as the thermal coating for the BAT and XRT radiators.

\section{Lessons Learned from and Status of BAT and XRT Radiators}

\section{A. Adhesion}

Figure 3 shows the BAT radiator before it was painted. The ribs form 36 pockets, which are $4.064 \mathrm{~cm}$ (1.6 inch) deep. They are needed for structural reasons. In March 2001 a contract (NASA/GSFC contract P.O. No. S-48249G) was awarded to AZ-Tek to apply AZW-LA-II white paint to the BAT and XRT radiators at AZ-Tek. Initially the specifications for the paint in the contract were 0.09 for solar absorptance and 0.91 for emittance. They correspond to a paint thickness of $0.3302 \mathrm{~mm}$ (13-mil). Before the radiators were painted, the following exercises were performed. First, AZW-LA-II white paint was applied to a BAT radiator "pocket" sample. A tape lift test was performed after the paint was cured. It revealed that the paint had adhesion problems. The paint thickness around the bottom of the pocket and close to ribs was as much as $0.4572 \mathrm{~mm}$ (18 mil). It significantly exceeded AZ-Tek's $0.3302 \mathrm{~mm}$ (13- 
mil) thickness specification. On the contrary, the adhesion of a $15.24 \mathrm{~cm}(6$ inch $) \times 15.24 \mathrm{~cm}(6 \mathrm{inch})$ flat paint sample was good and the thickness met the specification.

Testing of AZW-LA-II paint samples at GSFC showed that adhesion problems occurred when the paint thickness exceeded $0.3302 \mathrm{~mm}(13 \mathrm{mil})$. To prevent adhesion problems, the radiator pocket paint thickness must be reduced. Solar absorptance versus thickness of AZW-LA-II was measured by AZ-Tek. Figure 4 presents the results. Reducing the thickness of AZW-LA-II to $0.1778 \mathrm{~mm}-0.254 \mathrm{~mm}$ ( 7 mil-10 mil) increases the solar absorptance only from 0.09 to 0.10 .

A new scheme was developed to solve the adhesion problem as follows: 1) apply AZ93 white paint primer to the radiator first, 2) after the primer is cured, mask a border around the ribs to prevent the AZW-LA-II white paint from building up at the pocket bottom when it is sprayed, 3) apply AZW-LA-II. As a result, the specifications of paint thickness on the pocket bottom in the contract to AZ-Tek were changed to $0.1778 \mathrm{~mm}-0.254 \mathrm{~mm}$ ( $7 \mathrm{mil}-10 \mathrm{mil})$. A paint thickness of $0.0762 \mathrm{~mm}-0.127 \mathrm{~mm}$ ( 3 mil-5 mil) on the ribs is needed to provide $0.1778 \mathrm{~mm}-0.254 \mathrm{~mm}$ ( 7 mil10 mil) on the pocket bottom. Also the specification for solar absorptance in the contract was increased to 0.10 , with a tolerance of \pm 0.01 .

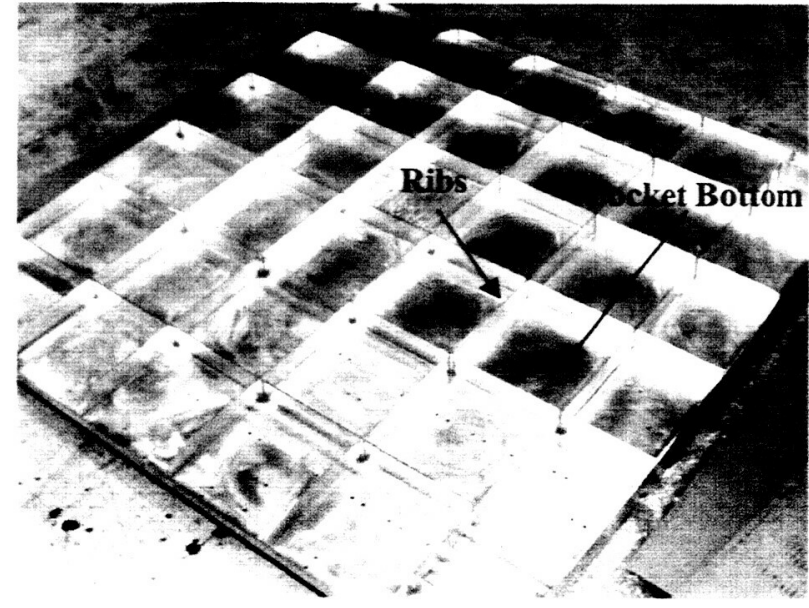

Figure 3. BAT Radiator Before It Was Painted.

Unlike the BAT radiator, the XRT radiator is flat and does not have any pockets. AZW-LA-II was applied to it first, while practice continued on the BAT radiator pocket sample. Application of AZWLA-II to the XRT radiator was completed at AZ-Tek in November 2001. The radiator and witness coupons were shipped to GSFC. Figure 5 shows the radiator when it was received.

\section{B. Qualification and Environmental Testing}

A qualification level thermal vacuum cycling test on AZW-LA-II white paint on the XRT radiator was performed at GSFC. It consisted of eight cycles from $-135^{\circ} \mathrm{C}$ to $-30^{\circ} \mathrm{C}$ and a hot survival exposure of $+35^{\circ} \mathrm{C}$. Figures 6 and 7 present the test results. The objective of the thermal vacuum test was to show the acceptability of AZW-LA-II white paint by

-Demonstrating that AZW-LA-II white paint on the radiator has good adhesion when the instrument is in an operating mode at temperatures in excess of the extremes predicted for the mission and during temperature transitions.

-Demonstrating that AZW-LA-II white paint on the radiator has good adhesion when the instrument is in an non-operating mode at cold and hot survival temperatures.

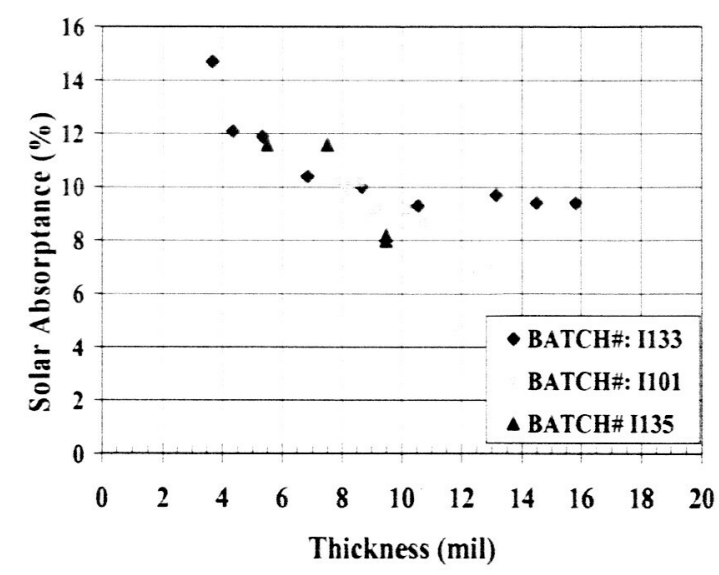

Figure 4. Solar Absorptance versus Paint Thickness.

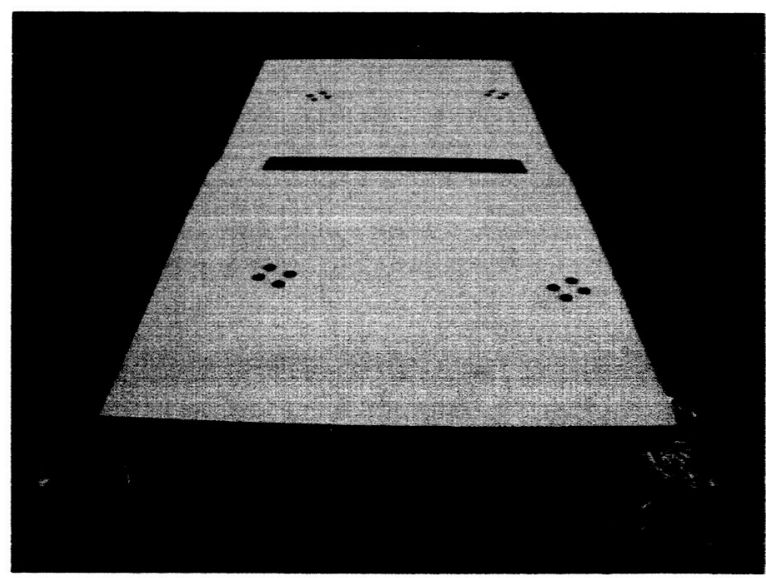

Figure 5. XRT Radiator AZW-LA-II White Paint. 


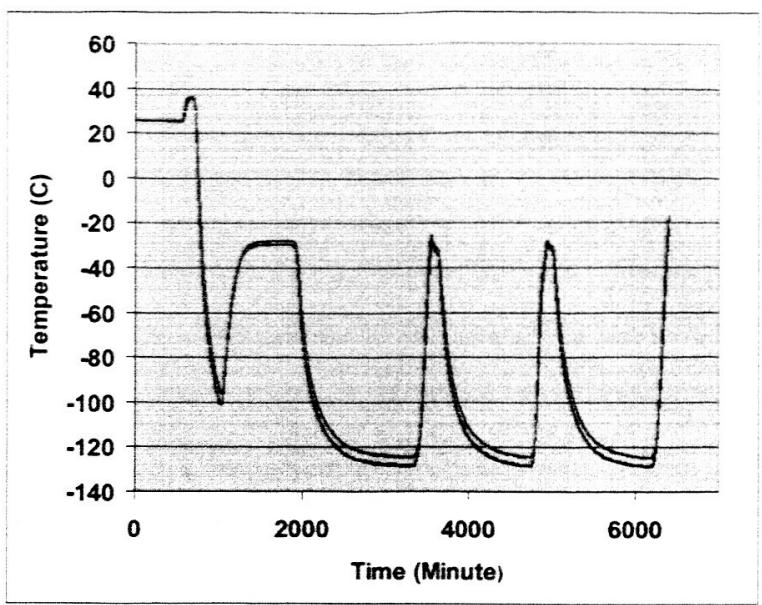

Figure 6. XRT Radiator Thermal Vacuum Cycling Data for Cycles 1-3.

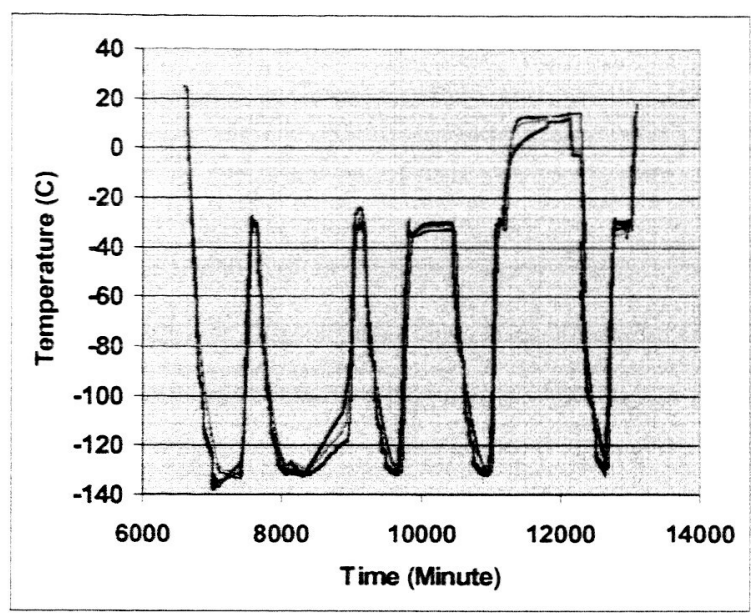

Figure 7. XRT Radiator Thermal Vacuum Cycling Data for Cycles 4-8.

Prior to the thermal vacuum cycling test, a small amount of AZW-LA-II came off the edges of the radiator. It was caused by handing of the radiator. A bakeout of the XRT radiator at $65^{\circ} \mathrm{C}$ was also performed during the XRT FPCA Heat Rejection System thermal performance verification test in a larger vacuum chamber. The total time in vacuum during these two tests is about 500 hours. Post-test inspections revealed that AZW-LA-II white paint remained in good condition. Because the white paint has passed the qualification test, the BAT radiator needed an acceptance test only.

Post thermal vacuum measurements on the eight XRT radiator witness coupons showed that the average solar absorptance is 0.11 , and the thickness of AZW-LA-II is $0.2032 \mathrm{~mm}$. $(8 \mathrm{mil})$.

Practices were made to paint the BAT radiator pocket sample using the aforementioned scheme at AZ-Tek. Inspections were made to verify that the paint adhesion was good, and the thickness of paint was measured to ensure that it met the specifications. After two successful practices, the BAT radiator and a pocket witness sample were painted at AZ-Tek in April 2002. They were shipped to GSFC after the paint was cured. Figure 8 illustrates the aforementioned coating scheme.

An acceptance level thermal vacuum cycling test was performed on the AZW-LA-II white paint of the BAT radiator, radiator pocket witness sample, and a $5.08 \mathrm{~cm}(2 \mathrm{inch}) \times 5.08 \mathrm{~cm}(2 \mathrm{inch})$ witness sample in a $2.1336 \mathrm{~m}(7$ $\mathrm{ft})$ diameter and $2.4384 \mathrm{~m}(8 \mathrm{ft})$ long vacuum chamber at GSFC in June 2002 . It consisted of four cycles from $-135^{\circ} \mathrm{C}$ to $+10^{\circ} \mathrm{C}$, and a hot survival exposure of $+35^{\circ} \mathrm{C}$. Figure 9 presents the test results. Post-test inspection revealed that the AZW-LA-II white paint on the radiator, pocket witness sample, and witness coupon remained in good condition. There was no adhesion problem. Following this test, twenty thermal vacuum cycles were added to the radiator pocket witness sample in a small vacuum chamber. The condition of AZW-LA-II did not change. The radiator was then transferred to bonded storage.

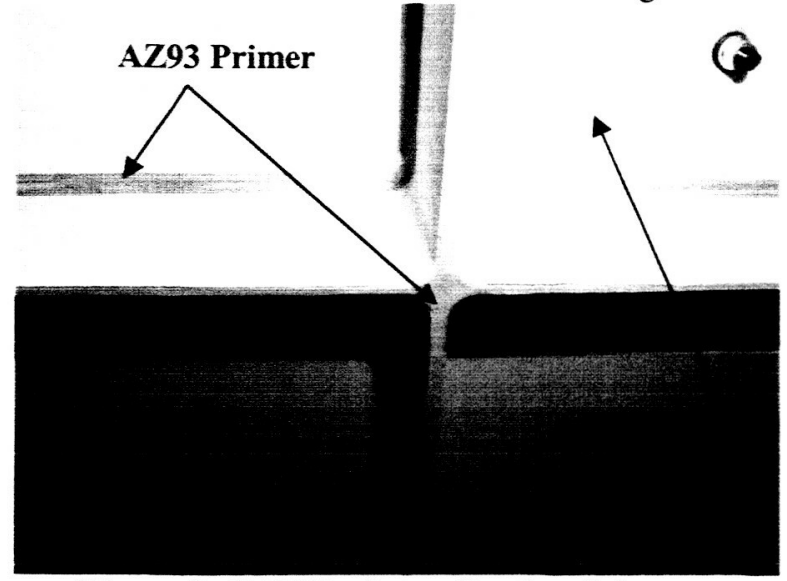

Figure 8. BAT Radiator Coating Scheme.

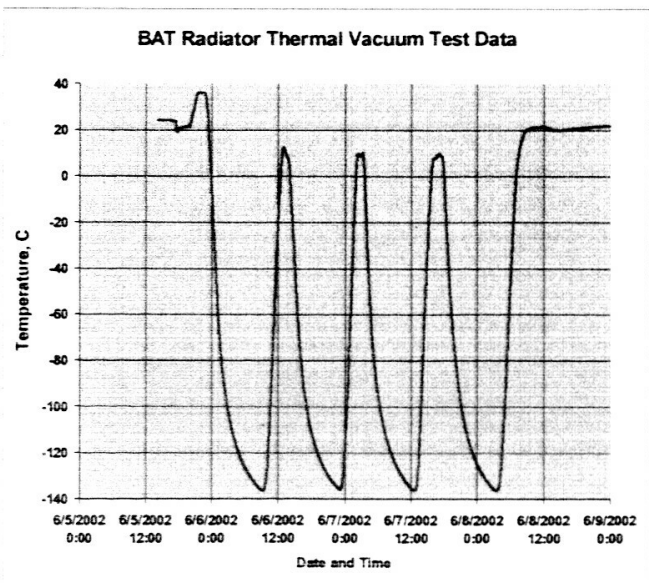

Figure 9. BAT Radiator Thermal Vacuum Cycling. 
Post thermal vacuum measurements on the BAT radiator pocket witness sample showed that the average solar absorptance is 0.11 , the emittance is 0.92 , and the thickness is $0.2032 \mathrm{~mm}(8 \mathrm{mil})$ to $0.2286 \mathrm{~mm}$ ( $9 \mathrm{mil}$ ). In December 2002 the BAT radiator was removed from storage. LHP condensers were integrated to the radiator in a clean tent at GSFC. Figure 10 shows the radiator and LHP assembly.

An inspection of the BAT radiator revealed that a small amount of the AZW-LA-II white paint along the edges had come off, and there were microcracks. An independent inspection by the Thermal Coatings Committee confirmed that handling caused the paint to come off, and air dryness caused microcracks. They were not concerned, and they recommended a touch up after environmental testing.

In January 2003, a thermal performance test was performed on the assembly of LHPs and radiator in a vacuum chamber at GSFC. Following this test, the assembly was integrated to the BAT instrument in March 2003. An instrument level mechanical vibration test was performed. A thermal balance test was performed on the BAT instrument in a vacuum chamber in June 2003. The total time in vacuum was nearly 350 hours. A thermal vacuum cycling test was performed on the BAT instrument in October 2003. The total time in vacuum was nearly 250 hours. The AZW-LA-II white paint remained in good condition after these environmental tests.

After the BAT and XRT were integrated to the Swift observatory, an observatory level mechanical vibration test was performed, followed by a thermal balance-thermal vacuum test from May 14 to June 22, 2004. The total time in vacuum is nearly 850 hours. The AZW-LA-II white paint on both radiators remains in good condition. Figure 11 shows the BAT and XRT radiators on the Swift observatory after environmental testing.

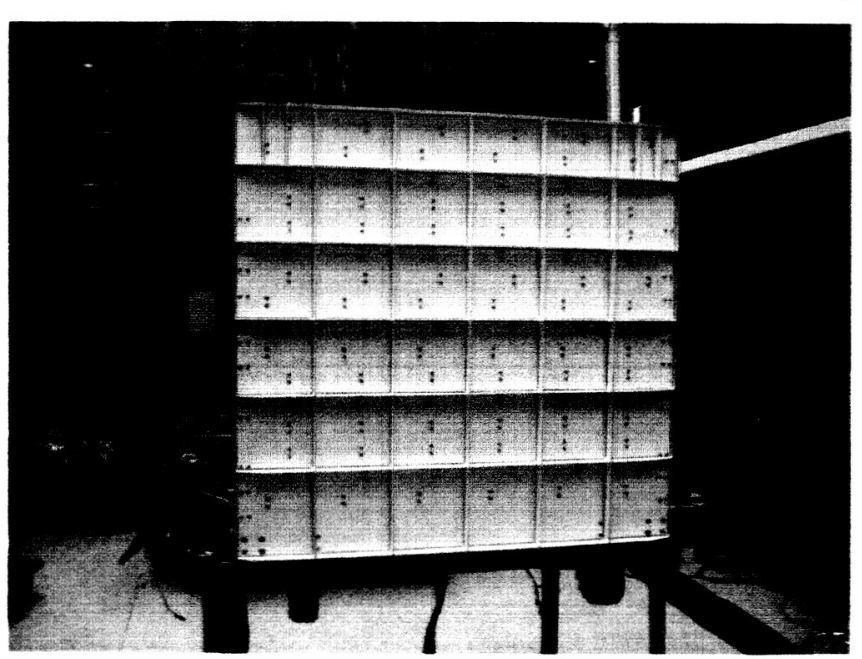

Figure 10. BAT Radiator AZW-LA-II White Paint.

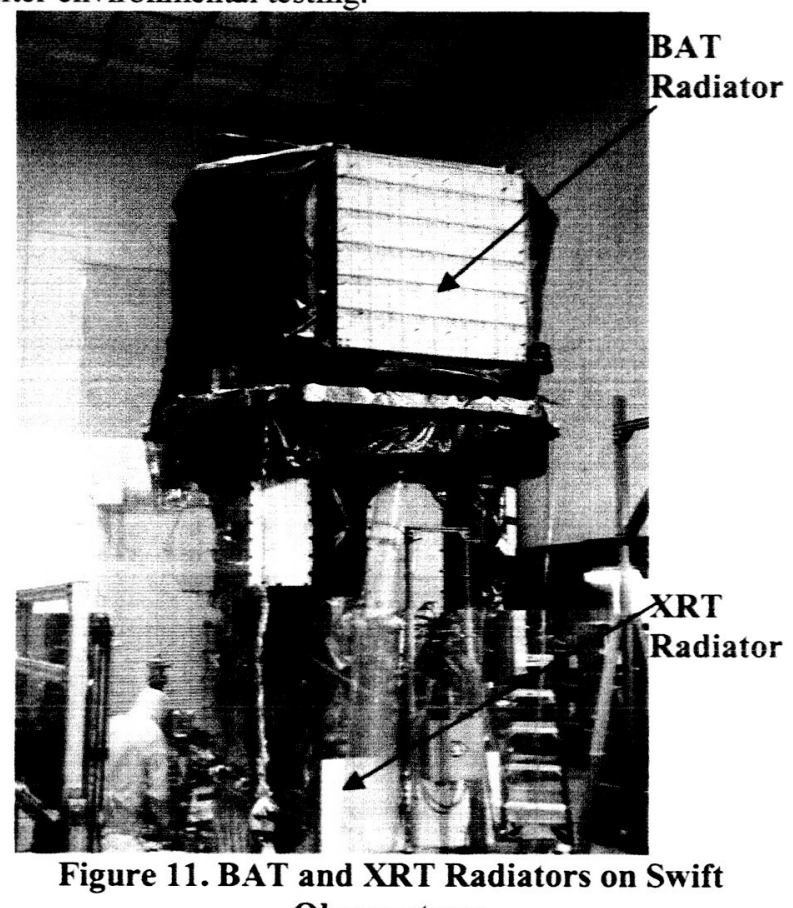

Observatory.

During the BAT instrument level and observatory level integration and testing from May 2003 through May 2004 , an air plenum cooling system was used to cool the BAT radiator. A plenum box was mounted to the radiator to enclose the white paint. Chilled air from an air conditioner was filtered by HEPA filters before it is supplied to the plenum box. The plenum box was installed and removed multiple times over the one-year period because the air plenum was not allowed under certain circumstances. For example, before moving the BAT instrument or Swift observatory from the clean tent to the vibration cell or vacuum chamber, the air plenum needs to be removed. Installation and removal of the air plenum box from the radiator added handling of the radiator. As a result, more AZW-LA-II white paint came off the edges of the radiator. Since the air plenum will be used to cool the BAT at the launch site, a touch up on the white paint won't be performed until the air plenum is removed from the radiator for the last time. Also vacuum cleaning will be performed on the BAT and XRT radiator at the launch site.

\section{Cost}

The total cost of applying AZW-LA-II white paint to the BAT radiator, a radiator pocket sample, and the XRT radiator was $\$ 131,900$ in 2002 . It included $\$ 40,000$ for the XRT radiator and $\$ 91,900$ for the BAT radiator and pocket sample. The cost per unit area of radiator was approximately $\$ 60,000 \mathrm{per} \mathrm{m}^{2}$. The major cost was for the 
paint materials. It is many times more expensive than white paints that have a higher solar absorptance. As mentioned earlier the paint thickness is $0.2032 \mathrm{~mm}(8 \mathrm{mil})$ for the XRT radiator, and $0.2032 \mathrm{~mm}(8 \mathrm{mil})$ to 0.2286 $\mathrm{mm}(9 \mathrm{mil})$ for the BAT radiator. The cosi increases as the paini thickness increases. If a thermal coating is required to minimize the effect of director solar or albedo flux or both in order to meet the thermal requirements, and cost is not an issue, AZW-LA-II should be considered. To prevent adhesion problems, the paint thickness should not exceed $0.3302 \mathrm{~mm}(13 \mathrm{mil})$.

\section{Summary and Conclusions}

AZ-Tek's AZW-LA-II white paint was previously flown as a flight experiment, and is being flown as a calorimeter. However it has never been flown as a thermal coating of radiators on an operational spacecraft before. This paint has the lowest solar absorptance among all white paints, and a very small degradation. But its cost is many times more expensive than white paints that have a higher solar absorptance. Results of adhesion test and ultraviolet exposure test at GSFC showed that AZW-LA-II is qualified for flight on Swift. To meet the thermal requirements of the BAT Detector Array and XRT FPCA CCD, it is used as the thermal coating for the BAT and XRT radiators. The lessons learned from flying this white paint as a thermal coating of radiators on an operational spacecraft for the first time are:

- Adhesion problems occurred when the paint thickness exceeded $0.3302 \mathrm{~mm}$ (13 mil).

- There was no problem of achieving a paint thickness of $0.3302 \mathrm{~mm}$ (13 mil) with a good uniformity for a flat radiator surface.

-For a radiator that has grids or ribs, such as the BAT radiator, the paint thickness needs to be significantly reduced in order to prevent adhesion problems.

-Reducing the paint thickness from $0.3302 \mathrm{~mm}(13 \mathrm{mil})$ to $0.2032 \mathrm{~mm}(8 \mathrm{mil})$ has solved the adhesion problem for the BAT radiator which has thirty-six $4.064 \mathrm{~cm}(1.6 \mathrm{inch})$ deep pockets.

-A reduction of the paint thickness from $0.3302 \mathrm{~mm}(13 \mathrm{mil})$ to $0.2032 \mathrm{~mm}(8 \mathrm{mil})$ increases the solar absorptance from 0.09 to 0.11 .

-The paint is rather fragile. Handling and shipping caused the paint to come off where contacts were made. Vacuum cleaning is needed to remove the white paint "dust".

-AZW-LA-II white paint on the XRT radiator remained in good condition after an 8-cycle qualification level thermal vacuum cycling test, and that on the BAT radiator remained in good condition after a 4-cycle acceptance level thermal vacuum cycling test.

-AZW-LA-II white paint on the BAT radiator pocket witness sample remained in good condition after 24 thermal vacuum cycles.

-AZW-LA-II white paint on both the XRT radiator and BAT radiator remain in good condition after additional temperature cycling in the observatory level thermal balance-thermal vacuum test.

-AZW-LA-II white paint on the XRT radiator has seen nearly 1,500 hours of high vacuum and remains in good condition, and that on the BAT radiator has seen nearly 1,800 hours of high vacuum and remains in good condition.

-AZW-LA-II white paint on the XRT radiator remained in good condition after the observatory level mechanical vibration test, and that on the BAT radiator remained in good condition after both the instrument level and observatory level mechanical vibration tests.

-AZW-LA-II white paint on the BAT radiator developed a number of microcracks after exposed to low relative humidity air in the clean tent. But they won't not cause the paint delaminate.

-Vacuum cleaning and paint touch up will be performed on AZW-LA-II of both the XRT and BAT radiators at the launch site, after the air plenum is removed from the BAT radiator for the last time.

\section{References}

'Choi, M. K., "Swift BAT Loop Heat Pipe Thermal System Characteristics and Ground/Flight Operation Procedure," Paper No. AIAA-2003-6077, $1^{\text {st }}$ International Energy Conversion Engineering Conference, Portsmouth, VA, Aug. 13-16, 2003.

${ }^{2}$ Choi, M. K., "Thermal Considerations of Swift XRT Radiator at $-35^{\circ} \mathrm{C}$ or Colder in Low Earth Orbit," Paper No. AIAA-20002906, IECEC 2000, Las Vegas, NV, July 24-28, 2000.

${ }^{3}$ Choi, M. K., "Thermal Design to Meet Stringent Temperature Gradient/Stability Requirements of Swift BAT Detectors," Paper No. AIAA-2000-2905, IECEC 2000, Las Vegas, NV, July 24-28, 2000. 